\title{
Estimativa da digestibilidade aparente da matéria seca por meio de indicadores internos em eqüinos ${ }^{1}$
}

\section{Rosane Barros da Silva Stein ${ }^{2}$, Luiz Roberto Aguiar de Toledo ${ }^{3}$, Fernando Queiroz de Almeida $^{4}$, Paulo Henrique Mazza Rodrigues ${ }^{5}$, César Gonçalves de Lima ${ }^{3}$, Anderson Corassa ${ }^{6}$, Thiago Marques dos Santos ${ }^{7}$}

\author{
${ }^{1}$ Parte da dissertação de Mestrado apresentada à Faculdade de Zootecnia e Engenharia de Alimentos - USP, pela primeira autora. Projeto \\ de pesquisa financiado pela FAPESP. \\ 2 Zootecnista, MSc. Endereço: Rua Luiz Massari, 77, Jd. São Paulo, Limeira, SP \\ ${ }^{3}$ Faculdade de Zootecnia e Engenharia de Alimentos - USP. \\ ${ }^{4}$ Universidade Federal Rural do Rio de Janeiro. \\ ${ }^{5}$ Faculdade de Medicina Veterinária e Zootecnia - USP. \\ ${ }^{6}$ Pós-graduação em Zootecnia - UFV. \\ 7 Graduação em Medicina Veterinária - UFRRJ. Bolsista de Iniciação Científica PIBIC/CNPq.
}

RESUMO - Com o objetivo de comparar metodologias de estimativa do coeficiente de digestibilidade da MS (CDMS) em dietas para eqüinos, avaliaram-se o método de coleta total de fezes (CT) e o uso dos indicadores internos celulose indigestível (CELi), fibra em detergente ácido indigestível (FDAi) e cinza insolúvel em ácido (CIA). No ensaio de digestão, foram utilizadas 16 éguas adultas da raça Mangalarga Marchador, entre 3,5 a 18,4 anos de idade e 394,80 \pm 46,22 kg. As dietas, isoprotéicas e isocalóricas, foram compostas de feno de coastcross (Cynodon dactylon cv. coast cross) e concentrado na proporção 60:40. Adotou-se delineamento experimental em blocos casualizados com quatro repetições em cada dieta. A avaliação das estimativas do CDMS pelos indicadores foi feita por meio de um modelo considerando-se o viés, ou seja, a diferença entre CDMS estimado pelo indicador e pela CT. Não houve efeito da idade dos animais no CDMS; entre os indicadores avaliados, a FDAi foi o que apresentou os parâmetros mais acurados. Os indicadores não diferiram quanto à precisão. Contudo, quanto à robustez, as estimativas da FDAi e da CELi foram afetadas pelo consumo de MS e pelo CDMS, enquanto a CIA foi afetada apenas pelo CDMS. As taxas de recuperação dos indicadores foram de 98,49; 94,49 e 144,5\% para a FDAi, CELi e CIA, respectivamente, e apenas a da FDAi não diferiu de $100 \%$. Os indicadores foram classificados segundo a acurácia, a precisão e a robustez na seguinte ordem: FDAi, CIA e CELi.

Palavras-chave: digestibilidade aparente, eqüinos, indicadores

\section{Estimate of dry matter apparent digestibility with internal markers in horses}

\begin{abstract}
This research aimed to compare dry matter digestibility coefficient (DMDC) of horse diets by total feces collection (TC) and internal markers, indigestible cellulose (iCEL), indigestible acid detergent fiber (iADF) and acid insoluble ash (AIA). Sixteen adult mares of Mangalarga Marchador breed averaging from 3.5 to 18.4 years and $394.80 \pm 46.22 \mathrm{~kg}$ were assigned to a randomized block design with four replicates. Diets were formulated to be isoprotein and isoenergetic and composed of coastcross hay (Cynodon dactylon cv. Coast cross) and 60:40 concentrate. Evaluation of DMDC estimated by the markers was performed by a model considering the bias, i.e., the difference between DMDC estimated by marker and by TC. No effect of animal age on DMDC. Among the evaluated markers, iADF was more accurate. Regarding precision, no differences were observed among markers. Concerning robustness, dry matter intake and DMDC affected iADF and iCEL estimates, whereas only DMDC affected AIA estimate. Markers recoveries were $98.49,94.49$ and $144.5 \%$ for iADF, iCEL e AIA, respectively and, only iADF recovery did not differ from $100 \%$. Markers ranking concerning accuracy, precision and robustness were as follows: iADF, AIA and iCEL.
\end{abstract}

Key Words: apparent digestibility, equine, markers

\section{Introdução}

Estudos sobre indicadores internos têm sido realizados em ensaios de digestão com eqüinos com o objetivo de tornar as estimativas de digestibilidade aparente de nutrientes mais práticas, econômicas e menos laboriosas.
Os indicadores cinza insolúvel em ácido (CIA), fibra em detergente ácido indigestível (FDAi) e celulose indigestível (CELi) têm apresentado estimativas próximas às do método de coleta total de fezes e, portanto, são considerados potenciais nas avaliações de dietas para eqüinos.

Correspondências devem ser enviadas para: rbsstein@terra.com.br 
Em estudos de digestão com eqüinos utilizando a CIA como indicador interno, alguns autores (Cudderford \& Hugues, 1990; Pereira \& Queiroz, 1997; Miraglia et al., 1999; Araújo et al., 2000) encontraram estimativas dos coeficientes de digestibilidade aparente dos nutrientes semelhantes às obtidas com o método de coleta total de fezes, com percentuais de recuperação fecal do indicador próximos de 100\%. Penning \& Johnson (1983a) utilizaram a CIA e a CELi como indicadores para estimar a digestibilidade aparente da matéria orgânica em ovinos e recomendaram a utilização da CELi. Penning \& Johnson (1983b) também constataram que a FDAi é um indicador confiável para predição da digestibilidade da matéria orgânica em ovinos.

Sunvold \& Cochran (1991) compararam o uso dos indicadores CIA e FDAi, entre outros, com a coleta total de fezes na estimativa da digestibilidade da matéria orgânica de diferentes forrageiras e relataram diferenças entre as estimativas obtidas com a FDAi e a coleta total de fezes, enquanto, com a CIA, não houve diferença significativa na estimativa de digestibilidade aparente da matéria orgânica dos fenos de gramíneas. Ferret et al. (1999) utilizaram a FDAi como indicador interno em ensaios de digestão com ovinos e não recomendaram sua utilização como indicador, em razão da variação na taxa de sua recuperação fecal conforme o tipo de feno ingerido.

Oliveira et al. (2003) observaram que a FDNi e FDAi ocasionaram superestimativa dos coeficientes de digestibilidade da fração fibrosa de dietas com diferentes proporções de volumoso, ao passo que a CELi foi o indicador interno mais adequado para estimativas da digestibilidade aparente em eqüinos.

Objetivou-se com este trabalho comparar metodologias da coleta total de fezes e indicadores internos (FDAi, CELi e CIA) para estimativa de coeficientes de digestibilidade aparente da matéria seca em dietas para eqüinos.

\section{Material e Métodos}

O experimento foi conduzido no Setor de Eqüinocultura do Instituto de Zootecnia da Universidade Federal Rural do Rio de Janeiro. As análises químicas foram efetuadas nos Laboratórios de Bromatologia e das Agrárias do Departamento de Zootecnia da Faculdade de Zootecnia e Engenharia de Alimentos da Universidade de São Paulo.

Foram utilizadas 16 éguas da raça Mangalarga Marchador, com 3,5 e 18,4 anos de idade e 394,8 $\pm 42,66 \mathrm{~kg}$. Os animais foram alojados em baias individuais $(3,5 \mathrm{mx} 3,0 \mathrm{~m})$ com piso de cimento, sem cama, providas de comedouros para concentrado, volumoso e sal e bebedouro.
Os tratamentos utilizados para o ensaio de digestão consistiram de dietas, isoprotéicas e isocalóricas, compostas de concentrado e volumoso na proporção 40:60, formuladas com quatro diferentes níveis de substituição $(0,33$, 66 e $100 \%$ ) do milho desintegrado com palha e sabugo (MDPS) pelo farelo de vagem de algaroba (FVA).

O delineamento utilizado para o ensaio de digestão foi em blocos casualizados, com quatro repetições por tratamento, compostos de acordo com a idade dos animais: Bloco I: 3,5 a 5 anos; Bloco II: 5 a 9 anos; Bloco III: 13 a 17 anos; e Bloco IV: 17 a 18,4 anos.

O experimento teve duração de 26 dias e foi composto de um período de 21 dias para adaptação às dietas e outro de cinco dias para coleta de fezes. A quantidade de alimento fornecido aos animais foi estabelecida segundo recomendações do NRC (1989), visando atender às exigências nutricionais para a categoria. Inicialmente, na fase de adaptação, os animais receberam dietas equivalentes a 1,7\% do peso vivo, em quilograma de matéria seca ao ar (90\% MS). Em seguida, o alimento foi fornecido à vontade e o consumo foi avaliado individualmente, evitando-se sobras na fase de coleta e mantendo a proporção de $60 \%$ feno de coastcross (Cynodon dactylon cv. Coast cross) picado e $40 \%$ de concentrado.

A dieta foi fornecida três vezes ao dia, às 7, 13 e $18 \mathrm{~h}$. As composições percentual e bromatológica dos concentrados e do feno são descritas nas Tabelas 1 e 2, respectivamente.

As fezes foram recolhidas diretamente do piso, acondicionadas em baldes plásticos e pesadas nos seguintes horários: 13 h (manhã - 8 às 13 h), 18 h (tarde - 13 às $18 \mathrm{~h}$ ), $24 \mathrm{~h}$ (noite - 18 às $24 \mathrm{~h}$ ) e $8 \mathrm{~h}$ (madrugada - 24 às $8 \mathrm{~h}$ ). Cada conjunto de horários constituiu de um dia de coleta. Após homogeneização e pesagem, foram retiradas alíquotas de $10 \%$ da produção de fezes obtida no período.

Foram elaboradas amostras compostas de fezes dos períodos de coleta total (manhã, tarde, noite e madrugada), por animal e por dia, que, posteriormente, formaram uma amostra composta dos cinco dias de coleta. Dessa amostra, foram retiradas alíquotas de $200 \mathrm{~g}$, que foram secas em estufa de ventilação forçada a $65^{\circ} \mathrm{C}$, por 48 horas, e, posteriormente, trituradas em moinho com peneira de $1 \mathrm{~mm}^{2}$ para as análises laboratoriais.

A coleta parcial de fezes foi realizada uma vez por período (manhã, tarde e noite). Amostras de 150 a $250 \mathrm{~g}$ foram coletadas imediatamente após defecação, na superfície da massa fecal, evitando-se contato com o piso. A seguir, procedeu-se à formação de amostras compostas por animal, que foram secas e moídas conforme procedimentos adotados para as amostras da coleta total de fezes. 
Tabela 1 - Composição centesimal dos ingredientes nos concentrados

Table 1 - Ingredient composition (\%) of the experimental diets

\begin{tabular}{|c|c|c|c|c|}
\hline \multirow[b]{2}{*}{$\begin{array}{l}\text { Ingrediente } \\
\text { Ingredient }\end{array}$} & \multicolumn{4}{|c|}{$\begin{array}{c}\text { Nível de substituição } \\
\text { Replacement level }\end{array}$} \\
\hline & $0 \%$ & $33 \%$ & $66 \%$ & $100 \%$ \\
\hline $\begin{array}{l}\text { Rolão de milho (MDPS) } \\
\text { Corn cob meal (CCM) }\end{array}$ & 75,00 & 50,00 & 25,00 & 0,00 \\
\hline $\begin{array}{l}\text { Farelo de vagem de } \\
\text { algaroba (FVA) }\end{array}$ & 0,00 & 25,00 & 50,00 & 75,00 \\
\hline Mesquite pod meal (MPM) & & & & \\
\hline $\begin{array}{l}\text { Farelo de soja } \\
\text { Soybean meal }\end{array}$ & 7,28 & 6,72 & 6,64 & 3,32 \\
\hline $\begin{array}{l}\text { Farelo de trigo } \\
\text { Wheat meal }\end{array}$ & 14,73 & 14,11 & 15,85 & 19,07 \\
\hline $\begin{array}{l}\text { Calcário calcítico } \\
\text { Limestone }\end{array}$ & 1,99 & 3,17 & 1,51 & 1,61 \\
\hline $\begin{array}{l}\text { Sal comum } \\
\text { Salt }\end{array}$ & 1,00 & 1,00 & 1,00 & 1,00 \\
\hline Total & 100,00 & 100,00 & 100,00 & 100,00 \\
\hline
\end{tabular}

${ }^{1}$ Substituição do MDPS (milho desintegrado com palha e sabugo) por FVA (farelo de vagem de algaroba) no concentrado.

${ }^{1}$ CCM (Corn cob meal) replacement with MPM (Mesquite pod meal) in the diet.

Tabela 2 - Composição bromatológica do feno e dos concentrados com níveis de substituição do MDPS pelo FVA

Table 2 - Bromatological composition of hay and concentrate with substitution levels of CCM by MPM

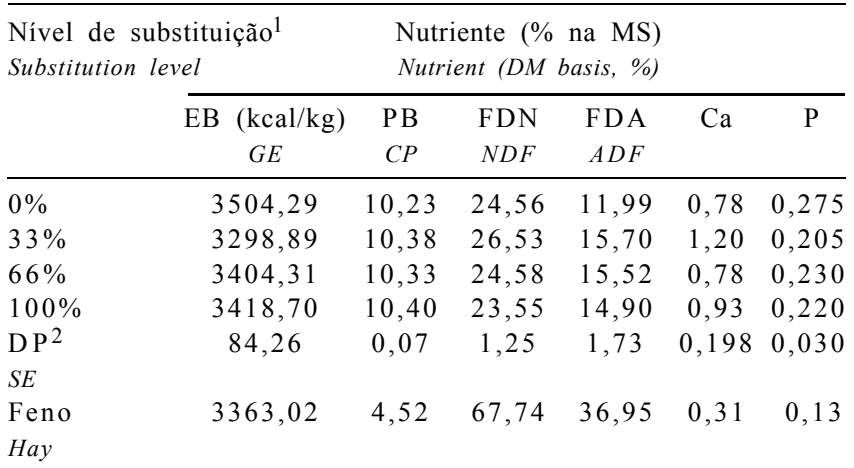

$\overline{1}$ Substituição do MDPS (milho desintegrado com palha e sabugo) por FVA (farelo de vagem de algaroba) no concentrado.

${ }_{1}^{1}$ CCM (Corn cob meal) replacement with MPM (Mesquite pod meal) in the diet

2 Desvio-padrão (Standard error).

Amostras de alimentos e sobras foram retiradas diariamente para posteriores análises pela mesma metodologia de coleta, pesagem, armazenamento, secagem e moagem utilizada para as fezes.

As análises bromatológicas dos alimentos, das sobras e das fezes foram realizadas segundo metodologia descrita por Silva \& Queiroz(2002). Os teores de FDAi e CELi foram obtidos após incubação in vitro das amostras em líquido ruminal, por 144 horas, conforme procedimentos descritos por Cochran et al. (1986). Os teores de CIA ( $\mathrm{HCl} 2 \mathrm{~N})$ foram determinados pela técnica relatada por Van Keulen \& Young (1977).

Para obtenção do consumo real dos nutrientes, foram consideradas as médias de consumo de feno e concentrado e das sobras para cada animal, segundo a equação:

$$
C R N=\frac{(C c \times N c+C f \times N f)-S \times N s}{100}
$$

em que $\mathrm{CRN}=$ consumo real de determinado nutriente $(\mathrm{g}$ de MS); $\mathrm{Cc}=$ quantidade de concentrado consumida ( $\mathrm{g}$ de $\mathrm{MS}) ; \mathrm{Nc}=$ concentração do nutriente no concentrado $(\% \mathrm{da}$ $\mathrm{MS}$ ); $\mathrm{Cf}=$ quantidade de feno consumida ( $\mathrm{g}$ de $\mathrm{MS}$ ); $\mathrm{Nf}=$ concentração do nutriente no feno ( $\%$ da MS); $\mathrm{S}=$ sobra média obtida no período de colheita ( $\mathrm{g}$ de $\mathrm{MS}$ ); $\mathrm{Ns}=$ concentração do nutriente nas sobras (\% da MS).

Os coeficientes de digestibilidade aparente da matéria seca estimados com o uso de indicadores (CDMS Ind) foram obtidos pelas seguintes equações:

$$
\text { CDMSInd }(\%)=100-100 \times(\mathrm{Ai} \div \mathrm{Afe})
$$

em que $\mathrm{Ai}=$ concentração do indicador ingerido (\% da MS); Afe $=$ concentração do indicador nas fezes ( $\%$ da MS). A concentração do indicador ingerido foi calculada da seguinte forma:

$$
\text { Ind.i }(g)=(A f \times C f)+(A c \times C c)-(A s \times S)
$$

em que Ind. $\mathrm{i}=$ quantidade do indicador ingerido ( $\mathrm{g}$ de MS); Af = concentração do indicador no feno (\% da MS); $\mathrm{Cf}=$ quantidade de feno consumida ( $\mathrm{g}$ de $\mathrm{MS}$ ); $\mathrm{Ac}=$ concentração do indicador no concentrado (\% da MS); $\mathrm{Cc}=$ quantidade de concentrado consumida (g de MS); As = concentração do indicador nas sobras (\% da MS); $\mathrm{S}=$ sobra média obtida no período de coleta ( $\mathrm{g}$ de MS).

A partir da concentração obtida, tem-se:

$$
\operatorname{Ai}(\%)=(\text { Ind. } \div \text { CMS }) \times 100
$$

em que $\mathrm{Ai}=$ concentração do indicador ingerido (\% da MS); Ind.i = quantidade do indicador ingerido ( $\mathrm{g}$ de $\mathrm{MS}$ ); CMS = consumo de MS (g de MS).

As equações utilizadas para estimativa da excreção fecal pelo indicador (Exc.) e sua taxa de recuperação (TR) foram:

$$
\text { Exc. }(g)=\frac{\text { Ind. } i}{\text { Ind.fe }} \quad \operatorname{TR}(\%)=\frac{\text { Ind.exc }}{\text { Ind. } i} \times 100
$$

em que Ind. $\mathrm{i}=$ quantidade do indicador ingerido ( $\mathrm{g}$ de MS); Ind.fe = concentração do indicador nas fezes (\% de MS); Ind.exc $=$ quantidade de indicador excretado ( $\mathrm{g}$ da MS).

A análise dos valores das estimativas de digestibilidade foi feita pela metodologia descrita por Jonker et al. (1998), utilizando o programa Statistical Analyses System (SAS, 1989), considerando-se as seguintes características dos dados:

viés - a diferença entre a estimativa do CDMS pelo indicador e estimativa do CDMS pela coleta total de fezes; 
acurácia - a capacidade de direcionar a atenção para aquilo que se quer observar. Está relacionado ao fato de observar o valor certo (IATROS, 2002) sendo mais acurado o indicador que possuir um viés médio próximo ao valor zero;

precisão - maior ou menor proximidade entre o valor observado e o valor estimado (IATROS, 2002). Está relacionado com o fato de acertar nas quantidades e, conseqüentemente, quanto menor os desvios padrões, observados para os vieses do indicador, mais preciso ele é;

robustez - caracterização do indicador menos influenciado por fatores alheios (peso, idade, dietas, consumo etc). Para que um indicador enquadre-se neste conceito, deve possuir, em relação às retas de regressão viés versus variáveis, pequenos coeficientes angulares e de determinação $\left(r^{2}\right)$, maiores erros-padrão e, ainda, a ausência de inclinação da reta indicada por altas probabilidades estatísticas.

As estimativas do modelo foram comparadas aos valores do viés (CDMS indicador - CDMS coleta total de fezes) do grupo de dados para cada indicador. As médias dos vieses e os respectivos desvios-padrão foram calculados e os vieses, submetidos à análise de variância, para avaliação da acurácia dos indicadores. Em seguida, aplicaram-se o teste Tukey, para comparação das médias, e o teste $t$, para averigüação de sua igualdade ao valor zero (viés $=0$ ), utilizando-se o PROC UNIVARIATE (SAS, 1989).

Para avaliação da precisão das estimativas dos indicadores, os desvios-padrão dos vieses foram submetidos ao teste de homogeneidade das variâncias (teste Levene) e a diferença entre eles foi analisada pelo teste t (SAS, 1989). As taxas de recuperação dos indicadores foram comparadas estatisticamente ao valor 100 , por meio do teste $t$, utilizando-se o PROC UNIVARIATE (SAS, 1989). A avaliação de robustez de cada indicador foi obtida utilizando-se o PROC GLM (SAS, 1989) pela regressão dos valores do viés, de acordo com os próprios valores de CDMS estimados pelo indicador e com outras variáveis selecionadas (dietas, peso, idade e consumo de matéria seca) que poderiam interferir nos resultados. Para comparação das inclinações das retas, aplicou-se o teste $t$-student. Todos os testes utilizados foram aplicados segundo Milliken \& Johnson (1984), a 5\% de probabilidade.

\section{Resultados e Discussão}

Os consumos médios das dietas foram de 6,72; 7,37; 6,57 e 7,43 kg MS/dia para os níveis de substituição de 0,33 , 66 e $100 \%$, respectivamente, do milho desintegrado com palha e sabugo pelo farelo de vagem de algaroba $(\mathrm{P}>0,05)$.
Não foram observadas diferenças nos CDMS entre as dietas experimentais $(\mathrm{P}>0,05)$. Os valores do CDMS e dos teores dos indicadores internos nas dietas podem ser observados nas Tabelas 3 e 4 .

Os resultados da avaliação dos indicadores, considerando-se a diferença da estimativa dos CDMS pelo indicador e pela coleta total de fezes, constam na Tabela 5 .

As medidas de acurácia dos indicadores CELi e FDAi, quanto à média dos vieses, não mostraram diferenças entre si pelo teste Tukey. No entanto, o indicador FDAi mostrou-se mais acurado por apresentar média de viés igual ao valor 0 (zero), não se diferenciando, conseqüentemente, da estimativa obtida pela coleta total. A média dos vieses da CIA mostrou-se significativamente diferente das obtidas com os demais indicadores $(\mathrm{P}<0,05)$, sendo, ainda, diferente de zero, caracterizando-se como o indicador menos acurado.

Penning \& Johnson (1983a), avaliando os indicadores CIA e CELi em ovinos alimentados com alfafa e ryegrass (Lolium perennecv. Melle) peletizados, utilizando o modelo descrito neste trabalho, compararam o CDMO obtido com os indicadores àqueles determinados pela coleta total de fezes e constataram que, com exceção da estimativa da CELi para o ryegrass, os vieses foram significativamente superiores a zero $(\mathrm{P}<0,05)$ e aqueles encontrados para CIA foram superiores àqueles obtidos pela CELi. Os autores atribuíram aos coeficientes de variação e às taxas de recuperação a alta variabilidade da CIA para predição do CDMO em animais recebendo alfafa, similar ao observado neste trabalho. A menor digestibilidade da alfafa poderia ocasionar redução na taxa de passagem, limitando o tempo necessário para esse indicador alcançar o equilíbrio no trato gastrointestinal.

Tabela 3 - Coeficientes de digestibilidade aparente da MS (\%) (CDMS) estimados pela coleta total de fezes (CT) e pelos indicadores CELi, FDAi e CIA de dietas com diferentes níveis de substituição do MDPS pelo FVA

Table 3 - $\quad$ Apparent digestibility coefficients of dry matter (\%) (DMDC) estimated by total feces collection (TC) and by internal markers $i C E L, I A D F$ and AIA of diets with different levels of CCM replacement with MPM

\begin{tabular}{lrrrr}
\hline $\begin{array}{l}\text { Nível de substituição (\%) } \\
\text { Replacement level (\%) }\end{array}$ & $\begin{array}{c}\mathrm{CT} \\
\mathrm{TC}\end{array}$ & $\begin{array}{c}\mathrm{FDAi}^{2} \\
\text { iADF }\end{array}$ & $\begin{array}{c}\mathrm{CELi}^{3} \\
\text { ICE }\end{array}$ & $\begin{array}{c}\mathrm{CIA}^{4} \\
\text { LAIA }\end{array}$ \\
\hline 0 & 51,53 & 51,03 & 47,62 & 65,24 \\
33 & 50,05 & 45,48 & 41,96 & 65,13 \\
66 & 47,66 & 49,48 & 47,14 & 66,26 \\
100 & 47,54 & 47,00 & 47,09 & 62,23 \\
$\mathrm{CV}(\%)^{5}$ & 4,86 & 4,53 & 6,62 & 2,70
\end{tabular}

${ }^{1}$ Substituição do MDPS (milho desintegrado com palha e sabugo) por FVA (farelo de vagem de algaroba) no concentrado.

${ }_{1}^{1}$ CCM (Corn cob meal) replacement with MPM (Mesquite pod meal) in the diet.

2 Fibra em detergente ácido indigestível (Indigestible acid detergent fiber).

${ }^{3}$ Celulose indigestível (Indigestible celullose).

${ }^{4}$ Cinza insolúvel em ácido (Acid insoluble ash)

5 Coeficiente de variação (\%) (Coefficient of variation). 
Tabela 4 - Teores de indicadores nas dietas, no feno e no concentrado (\% na MS) com diferentes níveis de substituição do MDPS pelo FVA

Table 4 - Markers contents in the diets, hay and concentrate (DM basis, \%), according to different levels of CCM replacement with MPM

\begin{tabular}{|c|c|c|c|c|c|c|}
\hline \multirow[t]{2}{*}{$\begin{array}{l}\text { Nível de substituição }(\%)^{1} \\
\text { Substitution level }\end{array}$} & \multicolumn{2}{|c|}{$\begin{array}{c}\mathrm{FDAi}^{2} \\
i A D F\end{array}$} & \multicolumn{2}{|c|}{$\begin{array}{c}\mathrm{CELi}^{3} \\
i C E L\end{array}$} & \multicolumn{2}{|c|}{$\begin{array}{c}\mathrm{CIA}^{4} \\
A I A\end{array}$} \\
\hline & $\begin{array}{l}\text { Concentrado } \\
\text { Concentrate }\end{array}$ & $\begin{array}{c}\text { Dieta }^{5} \\
\text { Diet }\end{array}$ & $\begin{array}{l}\text { Concentrado } \\
\text { Concentrate }\end{array}$ & $\begin{array}{c}\text { Dieta } \\
\text { Diet }\end{array}$ & $\begin{array}{l}\text { Concentrado } \\
\text { Concentrate }\end{array}$ & $\begin{array}{c}\text { Dieta } \\
\text { Diet }\end{array}$ \\
\hline 0 & 7,49 & 16,89 & 5,94 & 11,58 & 0,22 & 0,80 \\
\hline 33 & 11,64 & 18,64 & 8,31 & 12,58 & 0,20 & 0,79 \\
\hline 66 & 11,49 & 18,48 & 7,77 & 12,30 & 0,15 & 0,77 \\
\hline 100 & 14,41 & 19,84 & 8,67 & 12,77 & 0,15 & 0,78 \\
\hline Feno (Hay) & 23,56 & & 15,58 & & 1,21 & \\
\hline
\end{tabular}

1 Substituição na ração concentrada do MDPS (milho desintegrado com palha e sabugo) pelo FVA (farelo de vagem de algaroba).

${ }^{1}$ CCM (Corn cob meal) replacement with MPM (Mesquite pod meal) in the diet.

2 Fibra em detergente ácido indigestível (Indigestible acid detergent fiber).

${ }^{3}$ Celulose indigestível (Indigestible celullose).

4 Cinza insolúvel em ácido (Acid insoluble ash).

${ }^{5}$ Coeficiente de variação (\%) (Coefficient of variation).

Tabela 5 - Comparação das estimativas de CDMS por indicadores internos, por meio da análise do viés (CDMS indicador - CDMS coleta total de fezes)

Table 5 - Comparison of DMDC estimates by internal markers through bias analysis (marker DMDC - total feces collection DMDC)

$\begin{array}{llr}\text { Indicador } & \text { FDAi } & \text { CELi } \\ \text { Marker } & \text { CIA } & \text { iADF }\end{array}$

Médias do viés (\%) ${ }^{1,2}$ (Bias average)

Probabilidade (Probability)

Desvio-padrão ${ }^{3}$ (Standard deviation)

Probabilidade (Probability)

Probabilidade (Probability)
$-0,9475 b$
0,3598

4,0092

$<0,0500$

Acurácia (Accuracy)

$$
-3,2406 \mathrm{~b} *
$$

0,0221

15,5200 a *

0,0001

Precisão (Precision)

$\begin{array}{rr}5,0796 & 3,0737 \\ <0,0500 & <0,0500\end{array}$

Qualidade (Quality)

${ }_{1}^{1}$ Médias na mesma linha seguidas de diferentes letras são diferentes $(P<0,05)$ pelo teste Tukey.

1 Means within a row followed by different letters differ $(P<0.05)$ by Tukey test.

2 Probabilidade do teste $T$ de média $=0$. ${ }^{*}$ Média diferente de 0 (zero).

2 Probability of $T$ test of mean $=0 .{ }^{*}$ Mean different of $O$ (zero).

3 Teste de homogenidade das variâncias (Levene, $\mathrm{p}=0,0142$ ) seguido de teste $\mathrm{t}$.

3 Homogeneity of variance test (Levene, $p=0.0142$ ) followed by $T$ Test

4 Probabilidade do teste $T$ de média $=100$. ${ }^{\star *}$ Média diferente de $100(\mathrm{cem})$.

${ }^{4}$ Probability of $T$ test of mean $=100 .{ }^{* *}$ Mean different of 100 (one hundred).

Embora os coeficientes de digestibilidade da matéria estimados pela coleta total e CELi não tenham diferido, não se sabe se esses resultados poderiam ser aplicados a forragens verdes.

Comparando o viés da FDAi em relação à coleta total de fezes de ovinos alimentados com diferentes forrageiras (alfafa, ryegrass e trevo vermelho), Penning \& Johnson (1983b) concluíram que a FDAi é um indicador confiável para estimativa do CDMO, superando inclusive os indicadores CELi e CIA. Entretanto, outros autores relataram estimativas de CDMO com a FDAi diferentes da coleta total de fezes (Sunvold \& Cochran, 1991; Ferret et al., 1999).

Alvarenga et al. (1997), em estudo sobre o fluxo ileal de matéria seca, avaliaram diferentes indicadores, em eqüinos alimentados com dietas mistas ou compostas exclusivamente de volumosos. Esses autores observaram melhor recuperação da FDAi e concluíram que a FDAi foi o indicador que possibilitou melhores estimativas de fluxo ileal de MS nos eqüinos alimentados exclusivamente com feno. Oliveira et al. (2003) relataram que a FDAi foi adequada na estimativa dos coeficientes de digestibilidade da fração fibrosa de dietas para eqüinos com proporção volumoso:concentrado de 1:1, mas, em dietas com proporções diferentes, a CELi foi o indicador interno mais adequado para a estimativa da digestibilidade aparente dos nutrientes.

$\mathrm{Na}$ avaliação da precisão das estimativas de digestibilidade, compararam-se os desvios-padrão dos vieses dos indicadores, não sendo observadas diferenças 
significativas. Ressalta-se, entretanto, que o indicador é mais preciso quanto menor o desvio-padrão de seus vieses em relação aos da coleta total de fezes, a CIA pode ser considerado o mais preciso entre os indicadores avaliados.

A taxa de recuperação da FDAi não diferiu de $100 \%$ $(\mathrm{P}=0,4554)$, apesar da alta taxa de recuperação da CELi, com valor médio de $94,49 \%$. As estimativas de digestibilidade pela CIA foram, em média, 40\% acima daquelas calculadas pela coleta total de fezes, atribuindo-se estes resultados às maiores taxas de recuperação, de aproximadamente $144,5 \%$.

A literatura preconiza o teor mínimo de $0,75 \%$ (na MS) de CIA na dieta ou no alimento para que a estimativa da digestibilidade por este indicador, em ruminantes, seja precisa (Thonney et al., 1985, citado por Araújo, 1999). Sunvold \& Cochran (1991) atribuíram as superestimativas de CDMO e as altas taxas de recuperação da CIA, em novilhos de corte, ao baixo teor do indicador no feno de alfafa $(0,3 \%)$. Contudo, verificaram variação de acordo com a forragem utilizada (alfafa, bromegrass e feno de capim nativo).

Neste trabalho, apesar de o feno de coastcross apresentar alto teor de CIA e os concentrados baixos percentuais de CIA, a proporção ingerida na dieta total esteve adequada $(0,77$ a $0,80 \%)$.

Araújo (1999) registrou taxa de recuperação de CIA de 96,32,48 nas fezes de eqüinos alimentados com dieta composta por feno de coastcross e milho (60:40) e teor de CIA de $1,07 \%$ e ressaltou a importância do controle da contaminação da dieta por agentes que afetam a recuperação deste indicador. Neste ensaio, parte da dieta eventualmente derramada do cocho pelos animais pode ter sido ingeridacom areia e outras partículas do piso, afetando a ingestão e recuperação de CIA, o que, provavelmente, ocasionou as elevadas recuperações observadas.

Cuddeford \& Hugues (1990) e Miraglia et al. (1999) também observaram superestimativas dos coeficientes de digestibilidade pela CIA em relação à coleta total de fezes em eqüinos, embora não tenha havido diferenças significativas. Todavia, os resultados da literatura são contraditórios, observando-se ausência de superestimativa dos valores de digestibilidade e taxas de recuperação que se aproximaram de 100\% (Pereira \& Queiroz, 1997; Araújo, 1999; Araújo et al., 2000).

Na Tabela 6 encontra-se a avaliação da robustez de cada um dos indicadores. Os indicadores FDAi e CELi sofreram influência do CDMS e do consumo de matéria seca, enquanto a CIA, apesar de também ser afetada pelo CDMS, não foi influenciada pelo consumo de matéria seca $(\mathrm{P}=0,0902)$. $\mathrm{O}$ menor coeficiente angular da equação de regressão dos valores de CMS, de acordo com os valores dos vieses da CIA, comparado ao dos outros indicadores, reforça este resultado.

As variáveis peso vivo, idade e tratamento não interferiram nas estimativas de CDMS dos indicadores. Os resultados de robustez foram evidenciados pelas probabilidades estatísticas observadas quando realizadas as regressões dos valores dos vieses destes indicadores em função de cada uma das variáveis. A baixa probabilidade, isto é, o resultado significativo, indica a existência de inclinação da reta (alto coeficiente angular) e, portanto, que ela se desloca em função da variável, como pode ser observado nas Figuras 1 e 2.

As comparações entre as inclinações das retas das funções de regressão dos indicadores não foram significativas, comprovando que os indicadores CELi, FDAi e CIA são igualmente influenciados pelas variáveis CMS e CDMS. Quanto ao efeito do CDMS, a CELi apresenta, no entanto, maior coeficiente de determinação $\left(\mathrm{R}^{2}=0,7389\right)$, além do maior coeficiente angular entre as três retas, indicando maior interferência desta variável.

O coeficiente de determinação $\left(r^{2}\right)$ e o erro-padrão também demonstram a interferência da variável nas estimativas dos indicadores, pois, quanto menor o erro, menor a distribuição dos pontos ao longo da reta e, quanto mais elevado o coeficiente de determinação, maior a relação entre o viés e a variável e maior a interferência da variável nas estimativas dos coeficientes de digestibilidade.

Considerando os conceitos de acurácia, precisão e robustez, é possível pontuar falhas específicas dos indicadores, como o efeito do tipo de forragem/tratamento ou de variáveis que influenciam suas estimativas de digestibilidade. Desse modo, a aplicação progressiva deste modelo possibilitaria melhor conhecimento do real potencial do indicador.

O modelo de avaliação utilizado e desenvolvido neste trabalho é bastante inovador; nas condições deste trabalho e em concordância com outros autores (Penning \& Johnson, 1983b; Alvarenga et al., 1997), indica-se a FDAi como o melhor dos indicadores avaliados, seguido da CIA e da CELi. Nesta classificação considerou-se uma pontuação para cada quesito avaliado, de modo que a acurácia equivaleu a dois pontos, a precisão a um ponto, a qualidade a um ponto e a robustez a um ponto para cada variável (CDMS, CMS, idade, peso vivo e dieta), totalizando nove pontos. 
Tabela 6 - Avaliação da robustez dos valores dos coeficientes de digestibilidade por meio da comparação das equações de regressão obtidas a partir dos vieses dos indicadores (CDMS indicador - CDMS coleta total de fezes)

Table 6 - Robustness evaluation of digestibility coefficients values with comparation of equation regression obtained from markers biases (marker DMDC - total feces collection DMDC)

\begin{tabular}{|c|c|c|c|}
\hline \multirow[t]{2}{*}{$\begin{array}{l}\text { Variável } \\
\text { Variable }\end{array}$} & \multicolumn{3}{|c|}{$\begin{array}{c}\text { Indicador } \\
\text { Marker }\end{array}$} \\
\hline & $\begin{array}{l}\text { FDAi } \\
i A D F\end{array}$ & $\begin{array}{c}\text { CELi } \\
i C E L\end{array}$ & $\begin{array}{l}\text { CIA } \\
A I A\end{array}$ \\
\hline & \multicolumn{3}{|c|}{ CDMS (\%) (DMDC) } \\
\hline Coeficiente angular ${ }^{1,2}$ (Angular coefficient) & 0,9862 & 1,2104 & 0,6934 \\
\hline Erro-padrão (Standard error) & 0,2346 & 0,1952 & 0,2995 \\
\hline Probabilidade $^{3}$ (Probability) & \multicolumn{2}{|r|}{$\operatorname{CMS}(\mathrm{g})(D M I)$} & 0,0255 \\
\hline Coeficiente angular ${ }^{1}, 2$ (Angular coefficient) & $-2,2406$ & $-2,3833$ & $-1,4900$ \\
\hline Erro-padrão (Standard error) & 0,8590 & 0,8590 & 0,8590 \\
\hline $\mathrm{R}^{2}$ & 0,3645 & 0,2573 & 0,2747 \\
\hline Probabilidade $^{3}$ (Probability) & 0,0125 & 0,0082 & 0,0902 \\
\hline \multicolumn{4}{|c|}{ Dieta (Diet) } \\
\hline Coeficiente angular ${ }^{1,2}$ (Angular coefficient) & 0,0188 & 0,0540 & 0,0191 \\
\hline $\mathrm{R}^{2}$ & 0,0326 & 0,1669 & 0,0574 \\
\hline Probabilidade $^{3}$ (Probability) & 0,4929 & 0,0538 & 0,4858 \\
\hline \multicolumn{4}{|c|}{ Peso (kg) ( Weigh) } \\
\hline Coeficiente angular ${ }^{1,2}$ (Angular coefficient) & $-0,0159$ & $-0,0167$ & $-0,0287$ \\
\hline Erro-padrão (Standard error) & 0,0231 & 0,0231 & 0,0231 \\
\hline $\mathrm{R}^{2}$ & 0,0338 & 0,0232 & 0,1882 \\
\hline Probabilidade $^{3}$ (Probability) & 0,4948 & 0,4751 & 0,2213 \\
\hline \multicolumn{4}{|c|}{ Idade (anos) (Age (years) } \\
\hline Coeficiente angular ${ }^{1,2}$ (Angular coefficient) & 0,0593 & 0,0617 & $-0,0464$ \\
\hline Erro-padrão (Standard error) & 0,1793 & 0,1793 & 0,1793 \\
\hline $\mathrm{R}^{2}$ & 0,0084 & 0,0056 & 0,0086 \\
\hline Probabilidade $^{3}$ (Probability) & 0,7426 & 0,7327 & 0,7973 \\
\hline
\end{tabular}

${ }^{1}$ Coeficiente angular da equação $=a+b X$ e do viés (CDMS indicador - CDMS coleta total), conforme a variável.

1 Angular coefficient of the equation $=a+b X$ from bias (marker DMDC-feces total collect DMDC) in function of variable.

2 Comparação entre inclinações das retas pelo teste t-student $(P>0,05)$.

2 Comparation among line inclinations with $t$-student test $(P<0.05)$.

3 Probabilidade de a análise de variância indicar a inclinação da reta (teste $F$ ).

3 Probability of variance analysis to indicate the line inclination (F test).
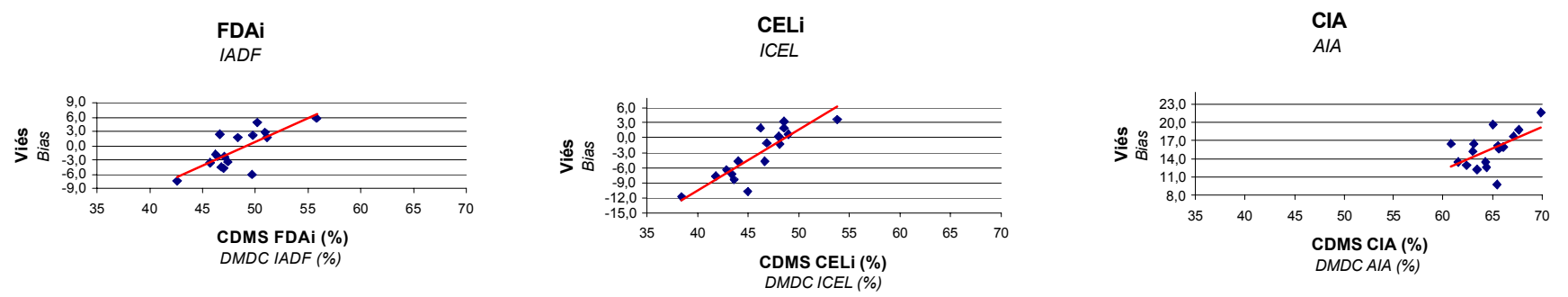

Figura 1 - Equações de regressão dos vieses dos indicadores (CDMS coleta total - CDMS indicador), conforme os CDMS estimados por cada indicador $(P=0,0001)$.

Figure 1 - Regression equations of the markers bias (DMDC total collection $-D M D C$ marker), as function of DMDC estimated by each one $(P=0.0001)$. FDAi (iADF): $\hat{Y}=-48,523+0,9862 X\left(r^{2}=0,5453\right)$; CELi (iCEL): $\hat{Y}=-58,862+1,2104 X\left(r^{2}=0,7389\right) ;$ CIA $(A I A): \hat{Y}=-29,337+0,6934 X\left(r^{2}=0,2813\right)$.

\section{Conclusões}

O modelo de avaliação de indicadores desenvolvido neste trabalho, considerando a diferença dos valores das estimativas pelo indicador e pela coleta total de fezes (viés), possibilitou a análise de fatores que afetam as estimativas de digestibilidade de indicadores, expondo seu real potencial de predição.
Além disso, como o modelo é simples, pode ser executado até mesmo na interpretação dos resultados, tendo como vantagem a padronização do método de avaliação dos indicadores, que torna as comparações entre os resultados de pesquisa mais verdadeiras.

O modelo possibilitou classificar os indicadores na seguinte ordem: FDAi, CIA e CELi, considerando-se a acurácia, precisão e robusteze, ainda, a taxa de recuperação. 

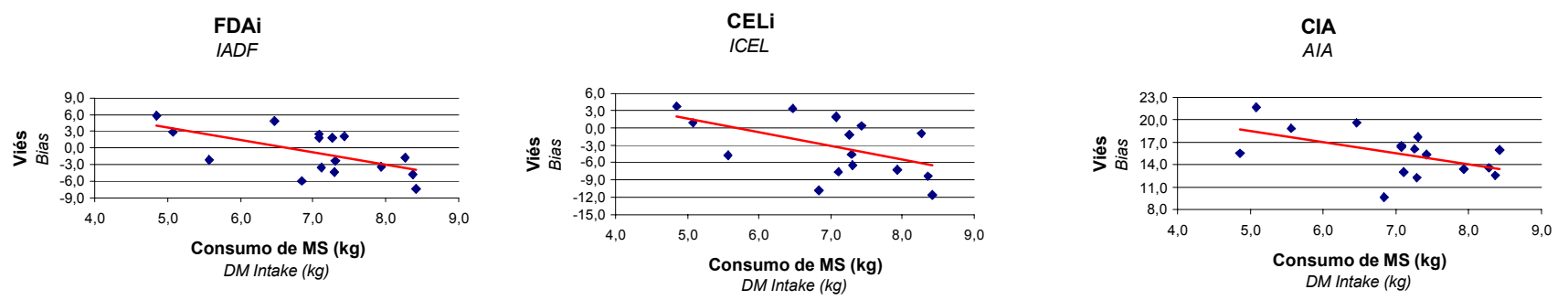

Figura 2 - Equações de regressão dos vieses dos indicadores (CDMS coleta total - CDMS indicador), conforme o consumo de matéria seca $(C M S)(P=0,002)$.

Figure 2 - Regression equations of the markers bias (DMDC total collection - DMDC marker) as function of dry matter intake $(D M I) P=0.002)$.

FDAi (iADF): $\hat{Y}=14,776-2,2391 x\left(r^{2}=0,3645\right)$; CELi (iCEL): $\hat{Y}=13,494-2,3832 x\left(r^{2}=0,2573\right)$; CIA (AIA): $\hat{Y}=25,987-1,4904 x\left(r^{2}=0,274\right)$.

\section{Agradecimento}

À FAPESP, pelo apoio e financiamento do projeto de pesquisa; à Universidade Federal Rural do Rio de Janeiro; e aos Professores Fernando Queiroz de Almeida, Paulo Henrique Mazza Rodrigues e César Gonçalves de Lima, que contribuíram com valiosas sugestões.

\section{Literatura Citada}

ALVARENGA, R.C.; LEÃO, M.I.; VALADARES FILHO, S.C. et al. Fluxo de matéria seca ileal e metodologias de coletas para amostragem da digesta em eqüinos fistulados. Revista Brasileira de Zootecnia, v.26, n.4, p.726-735, 1997.

ARAÚJO, K.V. Métodos para determinação da digestibilidade aparente dos nutrientes em eqüinos. Lavras: Universidade Federal de Lavras, 1999. 155p. Dissertação (Mestrado em Zootecnia) - Universidade Federal de Lavras, 1999.

ARAÚJO, K.V.; LIMA, J.A.F.; FIALHO, E.T. et al. Comparação entre os indicadores internos e o método de coleta total na determinação da digestibilidade dos nutrientes de alimentos volumosos, em eqüinos. Revista da Brasileira de Zootecnia, v.29, n.3, p.745-751, 2000.

COCHRAN, R.C.; ADAMS, D.C.; WALLACE, J.D. et al. Predicting digestibility of different diets with internal markers: evaluation of four potencial markers. Journal of Animal Science, v.63, p. $1476-1483,1986$.

CUDDEFORD, D.; HUGUIES, D. A comparison between chromiummordanted hay and acid-insoluble ash to determine apparent digestibitity of a chaffed, molassed hay/straw mixture. Equine Veterinary Journal, v.22, n.2, p.122-125, 1990.

FERRET, A.; PLAIXATS, J.; CAJA G. et al. Using markers to estimate apparent dry matter digestibility, faecal output and dry matter intake in dairy ewes fed Italian ryegrass hay or Alfafa hay. Small Ruminant Research, v.33, p.145-152, 1999.

IATROS: Estatística e pesquisa científica para profissionais de saúde. Acurácia e Precisão, 2002. Disponível em: http:// www.vademecum.com.br/iatros. Data: 02 de abril de 2002.

JONKER, J.S.; KOHN, R.A.; ERDMAN, R.A. Using milk urea nitrogen to predict nitrogen excretion and utilization efficiency in lactating dairy cows. Journal of Dairy Science, v.81, n.10, p.2681-2692, 1998.
MILLIKEN, G.A.; JOHNSON, D.E. Analysis of messy data: designed experiments. Belmont: Lifetime Learning Publication, 1984.

MIRAGLIA, N.; BERGERO, D.; BASSANO, B. et al. Studies of apparent digetibility in horses and the use of internal markers. Livestock Production Science, v.60, p.21-25, 1999.

NATIONAL RESEARCH COUNCIL - NRC. Nutrient requirements of horses. 5.ed.rev. Washington, D.C.: National Academy of Science, 1989. 100p.

OLIVEIRA, C.A.A.; ALMEIDA, F.Q.; VALADARES FILHO, S.C. et al. Estimativa da Digestibilidade aparente de nutrientes em dietas para eqüinos com o uso de óxido crômico e indicadores internos. Revista Brasileira de Zootecnia, v.32, n.6, p.16811689, 2003 (supl. 1).

PENNING, P.D.; JOHNSON, R.H. The use of internal markers to estimate herbage digestibility and intake: 1. Potentially indigestible cellulose and acid insoluble ash. Journal of Agricultural Science, v.100, p.127-131, 1983a.

PENNING, P.D.; JOHNSON, R.H. The use of internal markers to estimate herbage digestibility and intake: 2 . Indigestible acid detergent fiber. Journal of Agricultural Science, v.100, p.133-138, 1983b.

PEREIRA, J.C.; QUEIROZ, A.C. Digestibilidade aparente em eqüinos alimentados com capim elefante (Pennisetum purpureum Schum) e cana-de-açúcar (Saccharum officinarum L.) em diversas combinações. Revista Brasileira de Zootecnia, v.26, n.1, p.105-110, 1997.

SILVA, D.J.; QUEIROZ, A.C. Análise de alimentos: métodos químicos e biológicos. 3.ed. Viçosa, MG: Universidade Federal de Viçosa, 2002. 235p.

STATISTICAL ANALYSIS SYSTEMS - SAS. SAS/STAT ${ }^{\circledR}$ user's guide: version 6. 5.ed. Cary: 1989. v.2, 846p.

SUNVOLD, G.D.; COCHRAN, R.C. Technical note: evaluation of acid detergent lignin, alkaline peroxide lignin, acid insoluble ash, and indigestible acid detergent fiber as internal markers for prediction of alfafa, bromegrass, and prairie hay digestibility by beef steers. Journal of Animal Science, v.69, p.49514955, 1991.

Van KEULEN, J.; YOUNG, B.A. Evaluation of acid-insoluble ash as a natural marker in ruminant digestibility studies. Journal of Animal Science, v.44, n.2, p.282-287, 1977.

Recebido: 04/06/04 Aprovado:06/10/05 